\title{
Spin relaxation in charge-tunable InP quantum dots
}

\author{
Bipul Pal* and Yasuaki Masumoto \\ Institute of Physics, University of Tsukuba, Tsukuba 305-8571, Japan
}

(Received 16 June 2009; revised manuscript received 21 August 2009; published 29 September 2009)

\begin{abstract}
Spin relaxation in charge-tunable InP quantum dots was studied for quasiresonant excitation by means of both static and dynamic optical orientations. In neutral and one-electron-doped dots, spin relaxation of photogenerated electron-hole pairs takes place with a decay time of $\sim 45$ ps through phonon-mediated energy relaxation. In neutral dots, spin flip of single-particle holes additionally takes place and dark excitons are formed. Dark excitons work as spin reservoir and produce negative circular polarization under a small transverse magnetic field $(B)$. Spin orientation produced by optical pumping of the resident electrons in oneelectron-doped dots manifests negative circular polarization near $B=0$ and long coherence time of $1.7 \mathrm{~ns}$ for doped electron spins is observed. In two-electron-doped dots, spin dynamics is dominated by hole-spin relaxation in the lowest quantum state with a decay time of $\sim 50 \mathrm{ps}$.
\end{abstract}

DOI: 10.1103/PhysRevB.80.125334

PACS number(s): 78.67.Hc, 71.35.Pq, 71.35.Ji, 72.25.Fe

\section{INTRODUCTION}

Spin dynamics in semiconductors has attracted considerable attention of researchers in recent years for its possible application in spintronic devices. ${ }^{1}$ Especially, spins in quantum dots (QDs) are promising candidates for scalable quantum computers and quantum spin memory, ${ }^{2}$ because spinrelaxation processes are greatly suppressed by quantum confinement of carriers in all three spatial coordinates. ${ }^{3}$ Spin lifetime $e^{4,5}$ as well as spin coherence time ${ }^{6,7}$ much longer than the carrier recombination time have been experimentally demonstrated in charged QDs, where doped electrons or doped holes are present permanently and remove the limitation of radiative lifetime on spin lifetime of photogenerated carriers. Initialization, control, and readout of information in terms of spins have also been achieved for doped electrons or doped holes in QDs. ${ }^{8}$ Spin relaxation of electrons and holes in QDs critically depends on charging condition of QDs, because strong exchange interaction works among spins of electrons and holes. ${ }^{9}$ This means that spin dynamics in QDs can be manipulated by controlling its charge state. This can be very useful for spintronics applications where external controllability of spin dynamics is crucial. A thorough understanding of spin relaxation depending on the charging condition in QDs is needed both from physics point of view as well as for spintronics applications, but is at the elementary stage.

In this work, spin dynamics of electrons, holes, and electron-hole (e-h) pairs is studied under different charging conditions in charge-tunable InP QDs by means of timeresolved (TR) as well as time-integrated optical orientations ${ }^{10,11}$ for quasiresonant excitation at the excited state of the QDs, but below the wetting layer band gap. Besides resonant creation of spins, quasiresonant excitation is preferably used for spin injection in QDs when luminescence is used for the readout of spin information. ${ }^{12}$ In the latter case, spin relaxation through phonon-mediated energy relaxation is inevitable. Some studies reported that phononmediated energy relaxation diminishes spin memory almost completely, ${ }^{13}$ but our study showed that the spin memory is partially preserved by phonon-mediated energy relaxation. ${ }^{7}$
Present study aims at further clarification of the relationship between spin relaxation and phonon-mediated energy relaxation in QDs under different charging conditions. By using a combination of dynamic as well as static optical orientations we provide definitive knowledge on spin relaxation of electrons, holes, and correlated e-h pairs for one-electron (1e) doped, two-electron (2e) doped, and neutral QDs. Spin dephasing of doped electrons (1.7 ns) and that of correlated $\mathrm{e}-\mathrm{h}$ pairs (45 ps) dominate spin dynamics in 1e-doped QDs; relaxation of hole-spins (50 ps) at the ground state determines spin dynamics in 2e-doped QDs; and dark excitons play the most important role in spin dynamics of neutral QDs. In each case, spin relaxation takes place simultaneously with phonon-mediated energy relaxation but is not complete during energy relaxation.

\section{EXPERIMENTS}

The sample consists of self-assembled InP QDs grown by gas-source molecular-beam epitaxy on an $n^{+}$-GaAs substrate. ${ }^{14,15}$ A single layer of InP QDs with a nominal thickness of 4 monolayers is grown between the $100 \mathrm{~nm}$ $\mathrm{In}_{0.5} \mathrm{Ga}_{0.5} \mathrm{P}$ barrier layers. The average lateral diameter of the lens-shaped QDs is $40 \mathrm{~nm}$, and their height is $5 \mathrm{~nm}$. The areal density of the QDs is about $10^{10} \mathrm{~cm}^{-2}$. The sample shows, under above barrier excitation, a photoluminescence (PL) band peaked at $1.728 \mathrm{eV}$, as is seen in the right inset of Fig. 1. Because the lowest electron level in the QDs is close to the Fermi level of the doped substrate, an external electric bias $\left(U_{b}\right)$ allowed us to control the charged state of the QDs in the sample. ${ }^{16}$ A semitransparent indium-tin oxide Schottky contact on the top surface and a Au:Ge Ohmic contact on the back surface of the sample are deposited for the application of external electric bias. From previous studies of trionic quantum beat, ${ }^{16}$ electron spin quantum beat, ${ }^{17}$ and brightdark exciton quantum beat ${ }^{18}$ on this sample we know that the QDs are doped by one electron on an average at $U_{b}=-0.1 \mathrm{~V}$, they are doped by two electrons on an average at $U_{b}=+0.5 \mathrm{~V}$, and they are mostly neutral at $U_{b}=-0.6 \mathrm{~V}$. Measurements are made at these applied biases and spin re- 


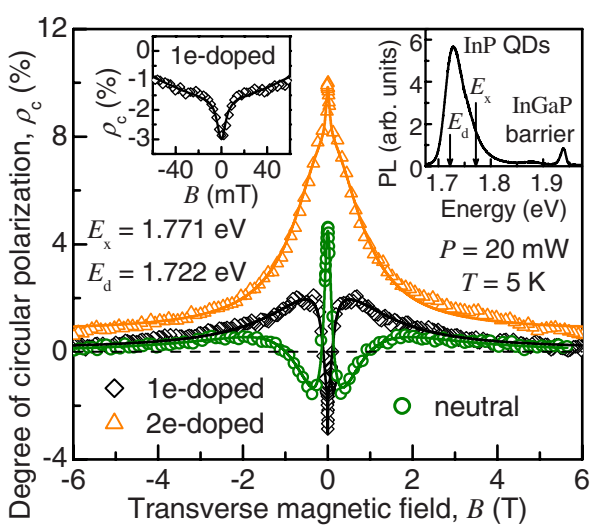

FIG. 1. (Color online) Hanle curves for three bias conditions for which QDs are either 1e doped $\left(U_{b}=-0.1 \mathrm{~V}\right)$, or $2 \mathrm{e}$ doped $\left(U_{b}=+0.5 \mathrm{~V}\right)$, or neutral $\left(U_{b}=-0.6 \mathrm{~V}\right)$. Solid lines are fits to the Hanle curves by the sum of two or three Lorentzians (discussed in the text). The left inset shows the expanded view of the very narrow portion of the Hanle curve near $B=0$ for 1e-doped QDs. The right inset shows the unpolarized PL spectrum for above barrier excitation of the sample under zero bias. Excitation and detection energies used in Hanle and PS-TR-PL measurements are indicated by arrows.

laxation in 1e-doped, 2e-doped, and neutral QDs is investigated.

Spin dynamics is studied by measuring the degree of circular polarization of PL,

$$
\rho_{c}=\frac{I_{S}-I_{O}}{I_{S}+I_{O}},
$$

where $I_{S}\left(I_{O}\right)$ is the intensity of the PL component having the same (opposite) circular polarization $\left(\sigma^{+}\right.$or $\left.\sigma^{-}\right)$as that of the excitation beam. ${ }^{10,11}$ We have used both polarizationselective (PS) TR-PL and Hanle effect measurements because they are complementary to each other. While PSTR-PL measurements can directly visualize the spin relaxation of electrons, holes, or e-h pair in real time, the Hanle effect can measure their spin relaxation in a clock of the Larmor precession of spin in a transverse magnetic field. Hanle effect is superior in sensitivity to the PS-TR-PL measurements. However, Hanle curves often show complicated shape and an unambiguous interpretation of Hanle curve is not always easy. In such cases, a combination of Hanle and PS-TR-PL measurements may be used to explain rich spin dynamics of carriers in semiconductors. Theory and experimental setup of Hanle and PS-TR-PL measurements are well documented in the literature. ${ }^{10,11}$

In Hanle experiments, $\rho_{c}$ is measured as a function of a transverse (perpendicular to the optical excitation axis) magnetic field $B$ (Voigt configuration). In the simplest case, the Hanle curve (a plot of $\rho_{c}$ as a function of transverse $B$ ) has a Lorentzian shape

$$
\rho_{c}(B)=\frac{\rho_{0}}{1+\left(B / B_{1 / 2}\right)^{2}},
$$

where $\rho_{0}$ is the amplitude of the Lorentzian and the halfwidth $B_{1 / 2}=\hbar /\left(g \mu_{B} T_{2}^{*}\right)$ of the Hanle curve is determined by the spin dephasing time $T_{2}^{*}$ of carriers $(g=$ Landé $g$ factor, $\mu_{B}=$ Bohr magneton). ${ }^{19}$ Our Hanle measurements are performed by using a standard Hanle experimental setup. ${ }^{10} \mathrm{We}$ excite the QDs quasiresonantly at the excitation energy $E_{x}=1.771 \mathrm{eV}$ along the sample growth axis by circularly polarized beam from a CW Ti:Sapphire laser. The excitation beam is focused to a spot of about $150 \mu \mathrm{m}$ in diameter on to the sample kept at $T \approx 5 \mathrm{~K}$ in a magneto-optical cryostat with a superconducting magnet $(|B| \leq 6 \mathrm{~T})$. We estimate that at the average excitation power $P=20 \mathrm{~mW}$ used here, less than one e-h pair per dot is photogenerated on an average. A photoelastic modulator modulates the circular polarization of the excitation beam between $\sigma^{+}$and $\sigma^{-}$polarizations at a frequency of $42 \mathrm{kHz}$. The circular polarization of groundstate PL is measured at the detection energy $E_{d}=1.722 \mathrm{eV}$ in the reflection geometry with a quarter wave $(\lambda / 4)$ plate and a Glan-Thompson linear polarizer (GTP) as the circular polarization analyzer. The PL is spectrally dispersed in a subtractive dispersion double monochromator, and an energy band of $16 \mathrm{meV}$ is allowed to pass through the exit slit of the monochromator to the detector. The signal is measured by a photomultiplier tube with a $\mathrm{GaAs}(\mathrm{Cs})$ photocathode and a two-channel gated photon counter. Because the spinrelaxation time of the nuclei is much longer than the modulation period $(23.8 \mu \mathrm{s})$ of our photoelastic modulator, the nuclear spins cannot follow the alternating spin polarization of the electrons and there is no dynamic nuclear polarization effect on the optical orientation of electrons and holes in our Hanle measurements. ${ }^{10}$

Time-resolved measurement of the polarized PL is performed in a standard setup ${ }^{11}$ by using a synchroscan streak camera for excitation by 2 ps pulses from a mode-locked Ti:Sapphire laser with a pulse repetition rate of $82 \mathrm{MHz}$. Use of a subtractive dispersion double monochromator allowed a time resolution of $9 \mathrm{ps}$ for the TR-PL setup. Excitation and detection energies, detection energy bandwidth, excitation spot size, average excitation power, and sample temperature for PS-TR-PL measurements are kept same as those used in the Hanle measurements. Excitation beam is circularly polarized by using a GTP and a $\lambda / 4$ plate in PS-TR-PL measurements. The components of PL having same and opposite circular polarization relative to the excitation polarization are measured separately by using a $\lambda / 4$ plate and a GTP as the circular polarization analyzer to obtain $\rho_{c}$. Though the excitation polarization remains constant in our PS-TR-PL measurements, the effect of dynamic nuclear polarization on our data is negligible because it is very small at $P=20 \mathrm{~mW}$ used here, as concluded from a previous study of nuclear spin effect on this sample. ${ }^{20}$

\section{RESULTS AND DISCUSSIONS}

Typical data of Hanle and PS-TR-PL measurements on 1e-doped, 2e-doped, and neutral QDs are shown in Figs. 1 and 2, respectively. Both the Hanle and PS-TR-PL data show different behavior for QDs with different charge state, suggesting strong dependence of spin dynamics on charging condition of QDs. Instead of a single Lorentzian, Hanle curves are showing complex shape depending upon charge 


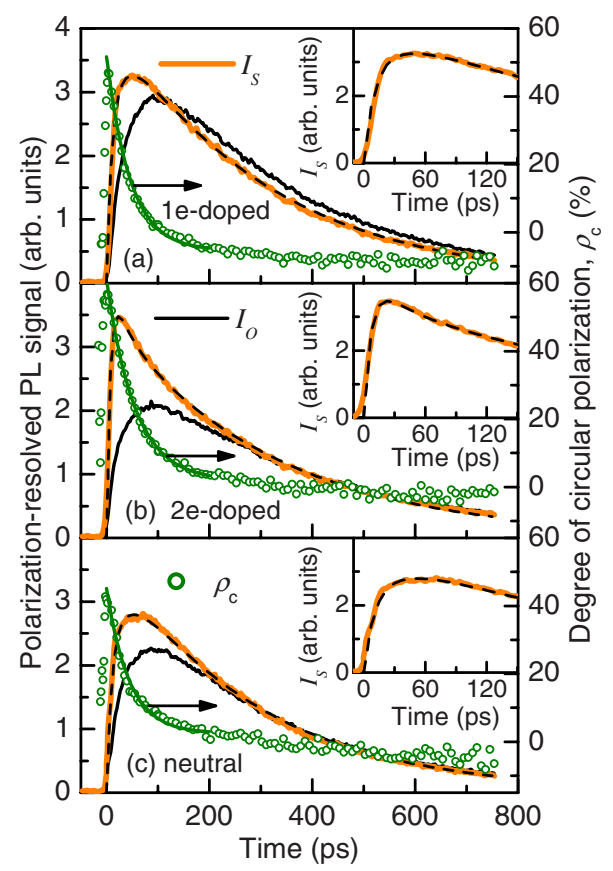

FIG. 2. (Color online) Time evolution of $I_{S}, I_{O}$, and $\rho_{c}$ for (a) 1e-doped, (b) 2e-doped, and (c) neutral QDs. Dashed lines are the fits (discussed in the text) to $I_{S}$. Initial part of $\rho_{c}$ in each case is fitted with an exponential decay (solid lines). Insets in (a), (b), and (c) show the expanded views of corresponding $I_{S}$. Here, $E_{x}=1.771 \mathrm{eV}, E_{d}=1.722 \mathrm{eV}, P=20 \mathrm{~mW}, T=5 \mathrm{~K}$, and $B=0 \mathrm{~T}$.

state of QDs. Such rich features in Hanle curve may arise due to spin relaxation of multiple species, e.g., electron, hole, or e-h pair, over different time scales. ${ }^{7,19}$ We fit (solid lines) the Hanle curves in Fig. 1 with sum of two or three Lorentzians of different amplitude $\left(\rho_{0}^{i}\right)$ and halfwidth $\left(B_{1 / 2}^{i}\right)$

$$
\rho_{c}(B)=\rho_{\infty}+\sum \frac{\rho_{0}^{i}}{1+\left(B / B_{1 / 2}^{i}\right)^{2}} .
$$

Each $B_{1 / 2}^{i}=\hbar /\left(g_{i} \mu_{B} T_{2 i}^{*}\right)$ is related to the spin dephasing time $T_{2 i}^{*}$ of a different species having a g-factor $g_{i}$. In the following we present a detailed analysis and interpretation of our data. A combination of Hanle and PS-TR-PL data helps us to identify various spin-relaxation processes in QDs with different charge states.

\section{A. One-electron-doped dots}

We have discussed the details of Hanle experimental data of 1e-doped InP QDs in a preceding paper. ${ }^{7}$ Here we briefly mention the main results. For 1e-doped QDs biased at $U_{b}=-0.1 \mathrm{~V}$, Hanle curve has three distinct regions (Fig. 1). It is very narrow near $B=0$ and $\rho_{c}$ is negative. An expanded view of the narrowest portion of the Hanle curve is shown in the left inset of Fig. 1. For $|B| \leq 0.5 \mathrm{~T}$ Hanle curve has a slightly broader negative dip, and for $|B|>0.5 \mathrm{~T}$ it has a broad positive peak. We use Eq. (3) to fit the Hanle curve with a sum of three Lorentzians with amplitudes of $-1.4 \%$, $-4 \%$, and $+2.3 \%$, and halfwidths of $4.5 \mathrm{mT}, 128 \mathrm{mT}$, and $1.53 \mathrm{~T}$, respectively. We ascribe the three Lorentzians with
$B_{1 / 2}=4.5 \mathrm{mT}, 128 \mathrm{mT}$, and $1.53 \mathrm{~T}$, respectively, to the spin dephasing of doped electrons constituting negative trions, correlated e-h pairs, and holes. ${ }^{7}$ It was found experimentally in this sample that for $B$ applied perpendicular to the sample growth direction we have for electrons, $g_{\perp \perp}=1.5$, independent of $B{ }^{17,18,21,22}$ However, for holes, experiments showed that in this sample the $\mathrm{g}$ factor nonlinearly decreases with increasing $B$, applied perpendicular to the sample growth direction, due to the mixing of different valence band states by the magnetic field. ${ }^{17,21}$ Decrease in hole g factor with increasing $B$ has been reported in the literature for InGaAs QDs. ${ }^{23}$ We obtain from Refs. 17 and 21 different values of hole g factor: $g_{\mathrm{h} \perp}=0.25(0.5)$ around $B=1 \mathrm{~T}(0.1 \mathrm{~T})$. With these values of electron and hole $\mathrm{g}$ factors, we get the spin dephasing time of doped electrons, correlated e-h pairs, and holes to be $1.7 \mathrm{~ns}, 45 \mathrm{ps}$, and $30 \mathrm{ps}$, respectively. The spin coherence time, $1.7 \mathrm{~ns}$, of the doped electrons is determined by the frozen fluctuation of nuclear spins in the QDs. ${ }^{7}$

We shall now analyze the PS-TR-PL data plotted in Fig. 2(a) for 1e-doped QDs. Inset of Fig. 2(a) zooms into the rise part of $I_{S}$. We see that the rise of $I_{S}$ has a fast and a slow component. Under quasiresonant excitation, e.g., by $\sigma^{+}$-polarized beam, an electron with up spin $\uparrow$ and a hole with down spin $\Downarrow$ are created at the excited state. Then the e-h pair goes down to the ground state. Energy relaxation by $41-57 \mathrm{meV}$ (a $16 \mathrm{meV}$ band around $1.722 \mathrm{eV}$ is detected) is mediated by the emission of a longitudinal optical (LO) phonon $(43.5 \mathrm{meV})$, a LO and an acoustic phonon, or cascade of acoustic phonons. In the process of energy relaxation by phonons, spin relaxation also takes place. Delayed rise of $I_{S}$ can be fitted by considering energy relaxation via both the LO and acoustic phonons

$$
I_{S}(t)=I_{0}\left[e^{-\gamma_{r} t}-\alpha e^{-\gamma_{l} t}-(1-\alpha) e^{-\gamma_{a} t}\right],
$$

where $\gamma_{r}$ is the e-h radiative recombination rate, $\gamma_{l}\left(\gamma_{a}\right)$ is the LO-phonon (acoustic phonon) -related energy relaxation rate, and $\alpha$ is the fractional contribution to energy relaxation by LO phonons. ${ }^{15,24}$ Using this fit [dashed line in Fig. 2(a)] we obtain ${ }^{25} \alpha=0.65, \quad \gamma_{r}=(305 \mathrm{ps})^{-1}, \quad \gamma_{l}=(9 \mathrm{ps})^{-1}$, and $\gamma_{a}=(33 \mathrm{ps})^{-1}$.

Time dependence of $\rho_{c}$ is displayed in Fig. 2(a) for 1edoped QDs. Major part of the decay of $\rho_{c}$ takes place within first 200 ps. Exponential fit to the initial part ${ }^{26}$ of the decay of $\rho_{c}$ results in a time constant of 45 ps which exactly matches with the spin dephasing time of correlated e-h pairs obtained above from the Hanle curve. This shows that spin dephasing of e-h pairs takes place during phonon-mediated energy relaxation, but it is slower than the energy relaxation. Beyond 120 ps (after the phonon-mediated energy relaxation is complete), $\rho_{c}$ becomes negative and it approaches a stable value of $-10 \%$ after $t \sim 300 \mathrm{ps}$. The negative $\rho_{c}$ in 1e-doped QDs is explained by considering both the optical pumping of the spins of doped electrons in QDs and simultaneous spin flip-flop process of photogenerated e-h pairs. ${ }^{4,27}$ The spin flip flop of e-h pair is caused by the strong anisotropic exchange interaction in the QDs. ${ }^{28}$ 


\section{B. Two-electron doped dots}

When two electrons are doped in a QD, they form a singlet state $" \sqrt{1 / 2}(\uparrow \downarrow-\downarrow \uparrow)$ " in the lowest electron quantum level and their spins are antiparallel to each other. Under $\sigma^{+}$ quasiresonant excitation a pair of an electron $\uparrow$ and a hole $\Downarrow$ is created at the excited state and the hole alone goes down to the ground state in 2e-doped QDs. Then the spin state of the photogenerated hole solely determines $\rho_{c}$, because the hole can recombine with one of the two doped electrons having antiparallel spins. Time evolution of $I_{S}$ for 2e-doped QDs biased at $U_{b}=+0.5 \mathrm{~V}$, has only a fast rise-component [Fig. 2(b) inset]. The slow component in rise of $I_{S}$ originating from acoustic phonon-mediated energy relaxation of electrons in 1e-doped QDs [Fig. 2(a) inset] is absent here. This means that the hole relaxation from the excited state to the ground state is much faster than the electron relaxation, as is believed. ${ }^{29}$ We fit [dashed line in Fig. 2(b)] the time evolution of $I_{S}$ for 2e-doped QDs with Eq. (4) by setting $\alpha=1$. The best fit yields $\gamma_{r}=(290 \mathrm{ps})^{-1}$ and $\gamma_{l}=(9 \mathrm{ps})^{-1}$.

The observed relaxation of $\rho_{c}$ in Fig. 2(b) is slower than the rise of $I_{S}$. This indicates that the relaxation of the spin polarization of holes at the lowest quantum state solely determines the decay of $\rho_{c}$ in 2e-doped QDs. Exponential fit to the initial part of time-resolved $\rho_{c}$ gives a decay time of 52 ps. The Hanle curve for 2e-doped QDs is positive in the entire range of $|B| \leq 6 \mathrm{~T}$ (Fig. 1). It is fitted with Eq. (3) by the sum of two Lorentzians. The major contribution to the fit comes from a Lorentzian of $\rho_{0}=7 \%$ and $B_{1 / 2}=0.913 \mathrm{~T}$. From $B_{1 / 2}=0.913 \mathrm{~T}$ we obtain $T_{2}^{*}=50 \mathrm{ps}$ using $g_{\mathrm{h} \perp}=0.25$ around $B=1$ T. ${ }^{17,21}$ It agrees well with the decay time of 52 ps obtained from PS-TR-PL data. Therefore we conclude that the spin relaxation of the holes takes place with a decay time of $\sim 50$ ps at the lowest quantum state in 2e-doped QDs.

\section{Neutral dots}

Under $\sigma^{+}$quasiresonant excitation a pair of an electron $\uparrow$ and a hole $\Downarrow$ is generated at the excited state in neutral QDs. Then both the electron and the hole relax to the ground state and form an exciton. Time evolution of $I_{S}$ for neutral QDs biased at $U_{b}=-0.6 \mathrm{~V}$, shows a fast component and a slow component [Fig. 2(c) inset], similar to the case of 1e-doped QDs [Fig. 2(a)]. This means that the energy relaxation involves both acoustic and LO phonons. We fit [dashed line in Fig. 2(c)] the time dependence of $I_{S}$ by Eq. (4) to obtain $\alpha=0.6, \quad \gamma_{r}=(280 \mathrm{ps})^{-1}, \quad \gamma_{l}=(10 \mathrm{ps})^{-1}$, and $\gamma_{a}=(34 \mathrm{ps})^{-1}$. Through the phonon-mediated relaxation, $\rho_{c}$ decreases with a decay time of $46 \mathrm{ps}$, as estimated from an exponential fit to the initial part of the decay of $\rho_{c}$. This decay time almost coincides with that in the 1e-doped QDs and is caused by the spin dephasing of correlated e-h pairs. The Hanle curve for neutral QDs has rich features. It is positive for small $(|B|<0.1 \mathrm{~T})$ as well as large $(|B|>1 \mathrm{~T})$ field, but becomes negative for $0.1<|B|<1$ T. It is fitted with Eq. (3) by a sum three Lorentzians. The major Lorentzian in the low field region has $\rho_{0}=7 \%$ and $B_{1 / 2}=108 \mathrm{mT}$. We use $g_{\mathrm{e} \perp}=1.5$ and $g_{\mathrm{h} \perp}=0.5$ around $0.1 \mathrm{~T}$ to obtain $T_{2}^{*}=53 \mathrm{ps}$, in agreement with the decay time obtained above from PS-TR-PL data. Strong anisotropic exchange interaction between the photo-

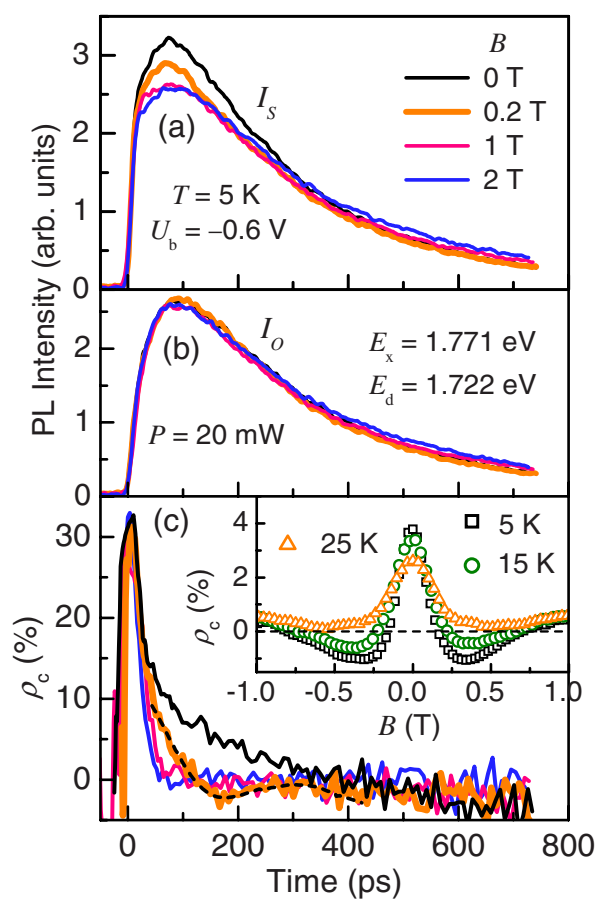

FIG. 3. (Color online) Time evolution of (a) $I_{S}$, (b) $I_{O}$, and (c) $\rho_{c}$ for neutral QDs under transverse magnetic field $B=0,0.2,1$, and 2 T. A smooth dashed curve in (c) is a guide to eyes to show a damped oscillatory behavior in the time evolution of $\rho_{c}$ for $B=0.2 \mathrm{~T}$. Temperature dependence of Hanle curve is plotted in the inset of (c).

generated e-h pair is responsible for efficient flip-flop type spin relaxation of the correlated e-h pair in neutral QDs, as is the case in 1e-doped QDs. ${ }^{28}$

One of the most intriguing features of the Hanle curve for neutral QDs is the negative $\rho_{c}$ region for $|B|=0.1-1 \mathrm{~T}$. To understand this we perform PS-TR-PL measurement under different transverse magnetic field. Data taken at $B=0,0.2$, 1 , and $2 \mathrm{~T}$ are plotted in Fig. 3 for illustration. We see that with increasing magnetic field in the low field range $(0<B<1 \mathrm{~T})$, time-resolved $I_{S}$ decreases for $t<200 \mathrm{ps}$. Beyond $B=1 \mathrm{~T}, I_{S}$ shows little decrease. In contrast, $I_{O}$ remains almost unaffected by the increase in $B$ in the range of $0-2 \mathrm{~T}$. This suggests formation of dark exciton and increase in their contribution with increasing $B$. Following considerations are needed to clarify the role of dark excitons in spin dynamics of neutral QDs. ${ }^{30}$

In addition to the spin relaxation of correlated e-h pair, spin flip of single-particle holes takes place during energy relaxation and leads to the formation of dark excitons $\uparrow \Uparrow$ in neutral QDs. ${ }^{31}$ Our previous study on the resonant spin orientation has clarified that the generation ratio between the bright and dark excitons is around 2:1 for the quasiresonant excitation at $1.771 \mathrm{eV}^{32}$ Without magnetic field, dark excitons are nonluminescent and do not contribute to $\rho_{c}$. However, a transverse magnetic field causes mixing between bright and dark excitons. Then dark excitons convert to bright excitons and luminesce. In this way they contribute to both time-resolved and time-integrated $\rho_{c}$. In the classical picture, transverse magnetic field induces the spin precession of an electron or a hole. Quantum picture of the spin preces- 
sion of an electron (a hole) is the mixing of the dark exciton $\uparrow \Uparrow$ and the bright exciton $\downarrow \Uparrow(\uparrow \downarrow)$.

The luminescence efficiency of bright excitons is intuitively given by the branching ratio: $\tau_{r}^{-1} /\left(\tau_{r}^{-1}+\tau_{0}^{-1}+\tau_{s}^{-1}\right)$, where $\tau_{r}\left(\tau_{0}\right)$ is the radiative (nonradiative) lifetime of excitons, and $\tau_{s}$ is the spin-flip time of either an electron or a hole, converting a bright exciton to a nonradiative dark exciton. In the reverse direction, dark excitons are converted to bright excitons at a spin-flip rate $\tau_{s}^{-1}$. After dark excitons are converted to bright excitons, they can luminesce. Therefore, we can consider the luminescence efficiency of dark excitons given by the product of the two branching ratios: $\tau_{s}^{-1} /\left(\tau_{s}^{-1}+\tau_{0}^{-1}\right)$ and $\tau_{r}^{-1} /\left(\tau_{r}^{-1}+\tau_{0}^{-1}+\tau_{s}^{-1}\right)$. With increasing $B$, spin-precession frequency increases and correspondingly $\tau_{s}$ decreases. With this, the luminescence efficiency of bright excitons decreases, but the luminescence efficiency of dark excitons increases in the low field region. The former explains the decrease in $\uparrow \Downarrow$ bright exciton PL and hence the decrease in $I_{S}$ in Fig. 3(a) with increasing $B$. The former also causes decrease in $\downarrow \Uparrow$ bright exciton PL. But it is compensated by the increase in $\uparrow \Uparrow$ dark exciton PL which comes predominantly through $\downarrow \Uparrow$ channel as discussed in the following paragraph. This explains the little change in $I_{O}$ with increasing $B$ in Fig. 3(b).

In InP QDs, transverse magnetic field as small as $0.2 \mathrm{~T}$ predominantly mixes $\uparrow \Uparrow$ with $\downarrow \Uparrow$, because $g_{\mathrm{e} \perp}=1.5$ is much larger than $g_{\mathrm{h} \perp}=0.25$. Precession period of an electron, $\tau_{s e}=h /\left(g_{\mathrm{e} \perp} \mu_{B} B\right)=238 \mathrm{ps}$, is less than $\tau_{r}=280 \mathrm{ps}$, while that of a hole, $\tau_{s h}=h /\left(g_{\mathrm{h} \perp} \mu_{B} B\right)=1.4 \mathrm{~ns}$, is much longer than $\tau_{r}$ and is comparable to $\tau_{0} \sim 10 \tau_{r}=2.8 \mathrm{~ns}$, for $B=0.2 \mathrm{~T}$. Then dark excitons $\uparrow \Uparrow$ predominantly emit $\sigma^{-}$PL. This is consistent with the decrease in $I_{S}$ and little change in $I_{O}$ with increasing $B$ in the low- $B$ range in Figs. 3(a) and 3(b). In time-integrated data (Hanle curve) this makes $\rho_{c}$ negative at small $B$.

On the other hand, large transverse magnetic field completely mixes $\uparrow \Uparrow$ with $\downarrow \Uparrow$ and $\uparrow \downarrow$, because $\tau_{s e}=24$ ps and $\tau_{s h}=143 \mathrm{ps}$ are shorter than $\tau_{r}$ and $\tau_{0}$ for $B=2 \mathrm{~T}$. Then dark excitons $\uparrow \Uparrow$ emit both $\sigma^{-}$and $\sigma^{+}$PL almost equally. This is consistent with the fact that beyond $1 \mathrm{~T}, I_{S}$ does not decrease with increasing $B$ and $\rho_{c}$ approaches zero in less than $100 \mathrm{ps.}$ This also explains the small increase observed for both $I_{S}$ and $I_{O}$ at long times ( $t>400 \mathrm{ps)}$ for large $B$ over the small field data in Figs. 3(a) and 3(b). Time-resolved $\rho_{c}$ for $B=0.2 \mathrm{~T}$ shows a damped oscillatory behavior which reflects the spin precession of an electron. An oscillation period of about 280 ps is estimated from this data, which approximately agrees with $\tau_{s e}=238 \mathrm{ps}$ at $B=0.2$ T. Dark excitons recombine nonradiatively and have long lifetime. They work as the spin reservoir and produce negative $\rho_{c}$ under the small transverse magnetic field. With the increase in temperature, thermal ac- tivation of spin flip increases the conversion from dark excitons to bright excitons and reduces the lifetime of dark excitons. As a result, the negative- $\rho_{c}$ structure in the Hanle curve between $0.1-1 \mathrm{~T}$ disappears above $25 \mathrm{~K}$, as is seen in the inset of Fig. 3(c). Broadening of the central Lorentzian with increasing temperature means that the spin dephasing time of correlated e-h pairs decreases at elevated temperature, as is expected.

Bracker et al. ${ }^{33}$ have studied spin dynamics in chargetunable GaAs QDs by Hanle measurements for negative and positive trions and neutral excitons. They used optical excitation in the quasicontinuum above the $Q D$ energy states. The photogenerated holes in their experiment lost their spin memory before getting captured into the QDs. As a result, neutral excitons showed almost no PL polarization and corresponding Hanle curve was featureless. For the same reason, Hanle curve for 1e-doped QDs (negative trions) showed only a single, very sharp Lorentzian, reflecting long spin dephasing time (16 ns) of doped electrons. Quasiresonant excitation in the QD excited state allowed us to study rich spin dynamics of e-h pair in neutral and 1e-doped QDs.

\section{CONCLUSIONS}

In conclusion, we study spin relaxation under quasiresonant excitation in charge-tunable InP QDs for different values of applied electric bias $\left(U_{b}\right)$ at which the QDs are either 1e-doped $\left(U_{b}=-0.1 \mathrm{~V}\right)$, or 2e-doped $\left(U_{b}=+0.5 \mathrm{~V}\right)$, or neutral $\left(U_{b}=-0.6 \mathrm{~V}\right)$. Comparison of data from complementary measurements such as Hanle effect and PS-TR-PL helps us to understand different spin-relaxation mechanisms depending on the charge state of the QDs. Long coherence time of $1.7 \mathrm{~ns}$ for doped electron spin is obtained in 1e-doped QDs, which is beneficial for spintronics application. In contrast, spin relaxation in 2e-doped QDs is rapid (50 ps) and it is determined by the hole-spin relaxation in the ground state. Dark excitons dominates the spin dynamics in neutral QDs and may act as spin reservoir. Spin relaxation of correlated e-h pair during phonon-mediated energy relaxation (33 ps) takes place through flip-flop process (45 ps) due to anisotropic exchange interaction in both 1e-doped and neutral QDs. Our data demonstrate external electric bias controlling of spin dynamics in InP QDs which is promising for spintronic devices.

\section{ACKNOWLEDGMENTS}

Preliminary study on the present topics was done together with S. Oguchi and M. Ikezawa whom authors acknowledge. This work was supported by Grant-in-Aid for Scientific Research No. 20244044 from the MEXT of Japan and R \& D promotion scheme funding international joint research promoted by NICT of Japan. 
*Present address: Indian Institute of Science Education and Research-Kolkata, Mohanpur, Nadia-741252, India; bipul@iiserkol.ac.in

${ }^{1}$ I. Žutić, J. Fabian, and S. Das Sarma, Rev. Mod. Phys. 76, 323 (2004).

${ }^{2}$ D. Loss and D. P. DiVincenzo, Phys. Rev. A 57, 120 (1998); P. Chen, C. Piermarocchi, and L. J. Sham, Phys. Rev. Lett. 87, 067401 (2001); Semiconductor Spintronics and Quantum Computation, Nanoscience and Technology, edited by D. D. Awschalom, D. Loss, and N. Samarth (Springer, Berlin, 2002).

${ }^{3}$ A. V. Khaetskii and Y. V. Nazarov, Phys. Rev. B 61, 12639 (2000); 64, 125316 (2001); L. M. Woods, T. L. Reinecke, and Y. Lyanda-Geller, ibid. 66, 161318(R) (2002).

${ }^{4}$ M. Ikezawa, B. Pal, Y. Masumoto, I. V. Ignatiev, S. Yu. Verbin, and I. Ya. Gerlovin, Phys. Rev. B 72, 153302 (2005); B. Pal, M. Ikezawa, Y. Masumoto, and I. V. Ignatiev, J. Phys. Soc. Jpn. 75, 054702 (2006).

${ }^{5}$ S. Cortez, O. Krebs, S. Laurent, M. Senes, X. Marie, P. Voisin, R. Ferreira, G. Bastard, J.-M. Gérard, and T. Amand, Phys. Rev. Lett. 89, 207401 (2002); T. Flissikowski, I. A. Akimov, A. Hundt, and F. Henneberger, Phys. Rev. B 68, 161309(R) (2003); M. Kroutvar, Y. Ducommun, D. Heiss, M. Bichler, D. Schuh, G. Abstreiter, and J. J. Finley, Nature (London) 432, 81 (2004); S. Laurent, B. Eble, O. Krebs, A. Lemaître, B. Urbaszek, X. Marie, T. Amand, and P. Voisin, Phys. Rev. Lett. 94, 147401 (2005).

${ }^{6}$ J. A. Gupta, D. D. Awschalom, X. Peng, and A. P. Alivisatos, Phys. Rev. B 59, R10421 (1999).

${ }^{7}$ Y. Masumoto, S. Oguchi, B. Pal, and M. Ikezawa, Phys. Rev. B 74, 205332 (2006).

${ }^{8}$ A. J. Ramsay, S. J. Boyle, R. S. Kolodka, J. B. B. Oliveira, J. Skiba-Szymanska, H. Y. Liu, M. Hopkinson, A. M. Fox, and M. S. Skolnick, Phys. Rev. Lett. 100, 197401 (2008); J. Berezovsky, M. H. Mikkelsen, N. G. Stolz, L. A. Coldren, and D. D. Awschalom, Science 320, 349 (2008); D. Press, T. D. Ladd, and Y. Yamamoto, Nature (London) 456, 218 (2008).

${ }^{9}$ A typical example is that the exchange interaction forces two identical electrons (holes) at the same quantum level to form a spin singlet.

${ }^{10}$ Optical Orientation, Modern Problems in Condensed Matter Sciences Vol. 8, edited by F. Meier and B. P. Zakharchenya (NorthHolland, Amsterdam, 1984).

${ }^{11}$ Spin Physics in Semiconductors, Springer Series in Solid-State Sciences Vol. 157, edited by M. I. Dyakonov (Springer, Berlin, 2008).

${ }^{12}$ D. Heiss, V. Jovanov, M. Bichler, G. Abstreiter, and J. J. Finley, Phys. Rev. B 77, 235442 (2008); D. Heiss, V. Jovanov, M. Caesar, M. Bichler, G. Abstreiter, and J. J. Finley, Appl. Phys. Lett. 94, 072108 (2009).

${ }^{13}$ S. Mackowski, T. A. Nguyen, T. Gurung, K. Hewaparakrama, H. E. Jackson, L. M. Smith, J. Wrobel, K. Fronc, J. Kossut, and G. Karczewski, Phys. Rev. B 70, 245312 (2004); Yu. G. Kusrayev, A. V. Koudinov, B. P. Zakharchenya, S. Lee, J. K. Furdyna, and
M. Dobrowolska, ibid. 72, 155301 (2005).

${ }^{14}$ H.-W. Ren, M. Sugisaki, S. Sugou, K. Nishi, A. Gomyo, and Y. Masumoto, Jpn. J. Appl. Phys., Part 1 38, 2438 (1999).

${ }^{15}$ I. V. Ignatiev, I. E. Kozin, V. G. Davydov, S. V. Nair, J.-S. Lee, H.-W. Ren, S. Sugou, and Y. Masumoto, Phys. Rev. B 63 075316 (2001).

${ }^{16}$ I. E. Kozin, V. G. Davydov, I. V. Ignatiev, A. V. Kavokin, K. V. Kavokin, G. Malpuech, H.-W. Ren, M. Sugisaki, S. Sugou, and Y. Masumoto, Phys. Rev. B 65, 241312(R) (2002).

${ }^{17}$ I. Ya. Gerlovin, I. V. Ignatiev, I. A. Yugova, and Y. Masumoto, Opt. Spectrosc. 104, 577 (2008).

${ }^{18}$ I. A. Yugova, I. Ya. Gerlovin, V. G. Davydov, I. V. Ignatiev, I. E. Kozin, H. W. Ren, M. Sugisaki, S. Sugou, and Y. Masumoto, Phys. Rev. B 66, 235312 (2002).

${ }^{19}$ R. I. Dzhioev, V. L. Korenev, B. P. Zakharchenya, D. Gammon, A. S. Bracker, J. G. Tischler, and D. S. Katzer, Phys. Rev. B 66 153409 (2002).

${ }^{20}$ B. Pal, S. Yu. Verbin, I. V. Ignatiev, M. Ikezawa, and Y. Masumoto, Phys. Rev. B 75, 125322 (2007).

${ }^{21}$ I. V. Ignatiev, T. Okuno, S. Yu. Verbin, I. A. Yugova, and Y. Masumoto, Physica E (Amsterdam) 17, 365 (2003).

${ }^{22}$ Y. Masumoto, I. V. Ignatiev, K. Nishibayashi, T. Okuno, S. Yu. Verbin, and I. A. Yugova, J. Lumin. 108, 177 (2004).

${ }^{23}$ A. Greilich, R. Oulton, E. A. Zhukov, I. A. Yugova, D. R. Yakovlev, M. Bayer, A. Shabaev, Al. L. Efros, I. A. Merkulov, V. Stavarache, D. Reuter, and A. Wieck, Phys. Rev. Lett. 96, 227401 (2006).

${ }^{24}$ Semiconductor Quantum Dots: Physics, Spectroscopy and Applications, Nanoscience and Technology, edited by Y. Masumoto and T. Takagahara (Springer, Berlin, 2002).

${ }^{25}$ The value of LO-phonon-mediated energy relaxation time $\gamma_{l}^{-1}$ $=9$ ps estimated from the fits in Fig. 2 is limited by the time resolution of our setup. Actual value of $\gamma_{l}^{-1}$ may be even smaller.

${ }^{26}$ Single exponential decay does not fit $\rho_{c}$ in the entire time range of measurement.

${ }^{27}$ I. V. Ignatiev, S. Yu. Verbin, I. Ya Gerlovin, R. V. Cherbunin, and Y. Masumoto, Opt. Spectrosc. 106, 375 (2009).

${ }^{28}$ K. V. Kavokin, Phys. Status Solidi A 195, 592 (2003).

${ }^{29}$ J. Shah, Ultrafast Spectroscopy of Semiconductors and Semiconductor Nanostructures, Springer Series in Solid-State Sciences, 2nd ed. (Springer, Berlin, 1999).

${ }^{30}$ K. C. Hall, E. J. Koerperick, T. F. Boggess, O. B. Shchekin, and D. G. Deppe, Appl. Phys. Lett. 90, 053109 (2007).

${ }^{31}$ Note that this discussion is based on $\sigma^{+}$excitation. The case of $\sigma^{-}$excitation can also be discussed by using the same logic.

${ }^{32}$ Y. Masumoto, K. Toshiyuki, T. Suzuki, and M. Ikezawa, Phys. Rev. B 77, 115331 (2008).

${ }^{33}$ A. S. Bracker, E. A. Stinaff, D. Gammon, M. E. Ware, J. G. Tischler, A. Shabaev, Al. L. Efros, D. Park, D. Gershoni, V. L. Korenev, and I. A. Merkulov, Phys. Rev. Lett. 94, 047402 (2005). 